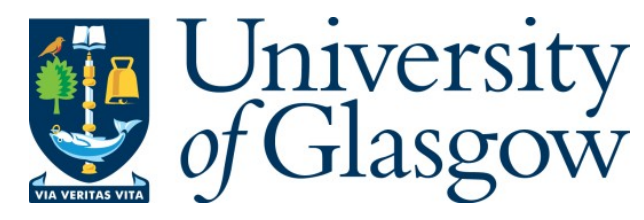

Silva, R. L., Secchin, L. D. , M oura-M elo, W. A. , Pereira, A. R., and Stamps, R. L. (2014) E mergence of skyrmion lattices and bimerons in chiral magnetic thin films with nonmagnetic impurities. Physical Review $B$, 89 (5). 054434 . ISSN 1098-0121

Copyright $\odot 2014$ A merican Physical Society

A copy can be downloaded for personal non-commercial research or study, without prior permission or charge

Content must not be changed in any way or reproduced in any format or medium without the formal permission of the copyright holder(s)

When referring to this work, full bibliographic details must be given

http://eprints.gla.ac.uk/94675/

Deposited on: 27 J une 2014

Enlighten - Research publications by members of the University of Glasgow http://eprints.gla.ac.uk 


\title{
Emergence of skyrmion lattices and bimerons in diluted chiral magnetic thin films
}

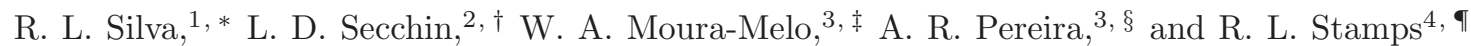 \\ ${ }^{1}$ Departamento de Ciências Naturais, Universidade Federal do Espírito Santo, São Mateus, 29932-540, Espírito Santo, Brazil \\ ${ }^{2}$ Departamento de Matemática Aplicada, Universidade Federal do Espírito Santo, São Mateus, 29932-540, Espírito Santo, Brazil \\ ${ }^{3}$ Departamento de Física, Universidade Federal de Viçosa, Viçosa, 36570-000, Minas Gerais, Brazil \\ ${ }^{4}$ SUPA School of Physics and Astronomy, University of Glasgow, Glasgow G12 8QQ, United Kingdom
}

(Dated: August 15, 2013)

\begin{abstract}
Skyrmions are topologically protected field configurations with particle-like properties that play important roles in various fields of science. Recently, skyrmions have been directly observed in chiral magnets. Here, we investigate the effects of nonmagnetic impurities (structural point-like defects) on the different initial states (random or helical states) and on the formation of the skyrmion crystal in a discrete lattice. By using first-principle calculations and Monte Carlo techniques, we have shown that even a small percentage of spin vacancies present in the chiral magnetic thin film affects considerably the skyrmion order. The main effects of impurities are somewhat similar to thermal effects. The presence of these spin vacancies also induces the formation of compact merons (bimerons) in both the helical and skyrmion states. We have also investigated the effects of adjacent impurities producing only one hole (with an almost circular shape) intentionally inserted into the plate (forming a non-simply connected manifold) on the skyrmion crystal.
\end{abstract}

PACS numbers: $75.10 . \mathrm{Hk}, 75.30 \mathrm{Ds}, 75.40 \mathrm{~Gb}, 75.40 \mathrm{Mg}$

\section{INTRODUCTION AND MOTIVATION}

Topological excitations are exciting objects in the physical world. In condensed matter physics, they frequently arise in the form of vortices and skyrmions which carry, in general, integer topological charges. However, topological excitations (more controversial) carrying only one-half of the skyrmion number, the so-called merons, may also be present in condensed matter systems. In this work, we pay attention to some topologically stable skyrmion and compact merons (bimerons) spin textures in diluted helical magnets. It is shown that some percentage of impurities introduced in these systems is able to induce the incidence of bimeron excitations and a deformation of the skyrmion lattice. The six fold order is lost for a very low concentration of spin vacancies.

Skyrmions were first found by Skyrme $\frac{1}{\underline{1}}$ about 50 years ago during an investigation of classical Lagrangian for possible models of subatomic particles as neutrons and protons (nucleons as discrete entities emerging from a continuous nuclear field). Despite skyrmion was originally introduced in field theory, it is now highly relevant to spin structures in condensed-matter systems as well. For example, such ideas were introduced in the context of the two-dimensional $(2 D)$ magnetism by Belavin and Polyakov ${ }^{2}(B P)$. These authors considered the continuum limit of the isotropic Heisenberg magnet, i.e., the nonlinear $\sigma$ model $(N L \sigma M)$, which is described by the Hamiltonian $\left.H_{\sigma}=J / 2 \int\left[\left(\partial_{\alpha} \vec{\mu}\right) \cdot \partial_{\alpha} \vec{\mu}\right)\right] d^{2} x$, where $J$ is the ferromagnetic coupling constant and $\mu=|\vec{\mu}|$ is the spin of the system (obeying the constraint $\vec{\mu}^{2}=$ $\mu_{x}^{2}+\mu_{y}^{2}+\mu_{z}^{2}=1$ ). In this framework, skyrmion-like objects show up as topological solitonic excitations carrying finite energy. Topologically, they correspond to the mapping of the spin-space sphere $\sum^{2}$ onto the continuum plane $\Re^{2}$. Their energy reads $E_{s}=4 \pi Q J \mu^{2}$, where $Q=\int\left(d^{2} r / 8 \pi\right) \epsilon_{i j} \epsilon_{\alpha \beta \gamma} \mu_{\alpha} \partial_{i} \mu_{\beta} \partial_{j} \mu_{\gamma}$ is the topological charge, counting the number of times the internal sphere $\sum^{2}$ is traversed as one span the physical space $\vec{r}=(x, y)$ (as compacted in a sphere). For $Q=1$, a $B P$ skyrmion of size $R$ has the well known configuration:

$$
\mu_{z}=\frac{r^{2}-R^{2}}{r^{2}+R^{2}}, \quad \mu_{x}+i \mu_{y}=2 i R \frac{x+i y}{r^{2}+R^{2}}
$$

Note that the skyrmion energy does not depend on the skyrmion size $R$, which is a signature of the scale invariance of the $N L \sigma M$.

Unfortunately, evidences for $B P$ skyrmions in layered isotropic classical magnets (not chiral) are only indirect. To our knowledge, the first indirect observation of such excitations in quasi- $2 D$ nearly classical magnets (a material with spin-5/2, described by the Heisenberg Hamiltonian) was reported by Waldner ${ }^{3.4}$ who found experimentally the skyrmion energy $\left(E_{\text {exp }}\right)$ from the heat capacity measurements in good agreement with theoretical prediction by $B P\left(E_{\exp } \simeq E_{s}\right)$. Further indirect observations of skyrmions were reported in quasi$2 D$ manganese compounds (systems with spin-5/2) and other class of isotropic classical antiferromagnets (propylammonium tetrachloromanganate also with spin-5/2), in which the skyrmion traces were found in the Electron Paramagnetic Resonance $(E P R)$ linewidth (see Zaspel et $a l \underline{\underline{5}})$. Such calculations were based on the skyrmionmagnon interactions which also resulted in an experimental skyrmion energy $E_{\text {exp }}$ simeq $E_{s}$. All these works (Waldner using a thermodynamic quantity and Zaspel et al. using a dynamic quantity) considered only indirect evidences for isolated skyrmions as excited states (in general, the isotropic Heisenberg magnets are expected to contain a diluted ideal gas of these excitations).

Recently, the discovery and real-space observations of the skyrmion phase of magnetization in magnetic mate- 
rials have renewed the interest in these objects in condensed matter physics, calling great attention of the physics community. Indeed, important direct experimental works 6.7 have undoubtedly revealed, for the first time, the very presence of skyrmions in chiral magnets, where the Dzyaloshinsky-Moriya $(D M)$ interaction plays a fundamental role (chiral materials are systems in which the mirror reflection symmetry is broken). However, differently from the above cases, in which isolated skyrmions (in a diluted gas of skyrmions) were indirectly considered in isotropic magnets, in the chiral magnets, they can be seen either in regular lattices or even as topologically stable single particles. Besides of being directly observed at low temperatures in films of $\mathrm{Fe}_{0.5} \mathrm{Co}_{0.5} \mathrm{Si}$ they also appear close to room temperature in $F e G e$. Actually, these compounds share the same $B 20$ structure with chiral cubic symmetry and they commonly show the helimagnetic ground state. Whenever a magnetic field is applied perpendicularly to the sample plane a $2 D$ skyrmion lattice emerges ${ }^{6}$. On the other hand, in iron films (with $D M$ interaction), skyrmions form spontaneously as the ground state, without necessity of being stabilized by any small external magnetic field ${ }^{7}$. Although the exact spin configuration of a single skyrmion in the skyrmion-lattice is somewhat different from that given in Eq.(1), the topological properties must remain basically the same, rendering the robustness of skyrmion-lattice as experimentally observed. Moreover, in a lattice, the skyrmion size $R_{L}$ is uniquely determined by the ratio of $D M$ and exchange energies (similarly, for skyrmions in the quantum Hall systems, the skyrmion size is fixed by the relative strengths of Zeeman and Coulomb interactions).

On the other hand, a meron-type excitation contrasts with typical topological defects (such as vortices) in easyplane magnets, once it carries half skyrmion number $\pm 1 / 2$ and vanishing vorticity. Thus, a pair of merons (a bimeron) is rather similar to a skyrmion, carrying integer skyrmion charge. The existence of merons in helical magnets is still less evident. Compact merons (or bimerons) have been proposed in the context of chiral magnets by Ezawa ${ }^{8}$. These objects are expected to arise above the ground state of these systems (the stripe helical state) whenever a stripe breaks into pieces as the magnetic field increases; the end points of a broken stripe bears opposite half skyrmion charges $( \pm 1 / 2)$ but the vortex number is zero. Thus, a finite stripe becomes a a pair of merons. The bimeron is then composed by two halfdisk domains and a rectangular stripe domain connecting these half-disks ${ }^{8}$. The bimeron is then composed by two half-disk domains and a rectangular stripe domain connecting these disks ${ }^{8}$. Consequently, the spin texture of a compact skyrmion could be obtained simply by removing the rectangular part of a bimeron and by joining the two half-disk domains. Ezawa ${ }^{8}$ has demonstrated that a treatment of merons as free particles qualitatively captures the phase diagram as predicted by Monte Carlo simulation 9 and observed in experiments ${ }^{6}$. Our results not only reinforce the existence of these compact merons

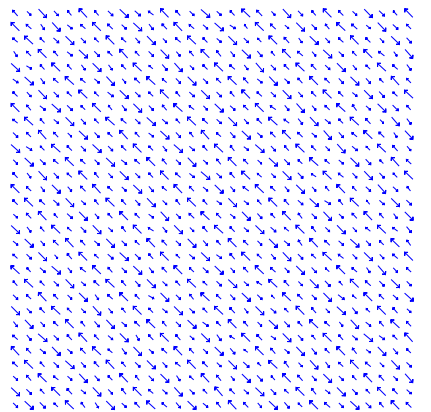

(a)

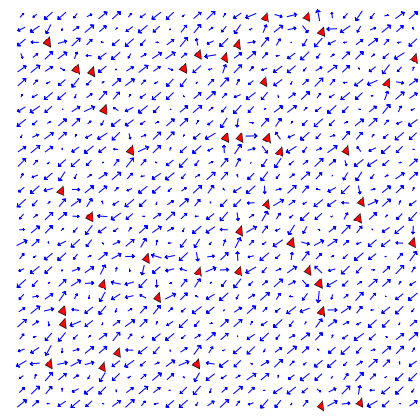

(c)

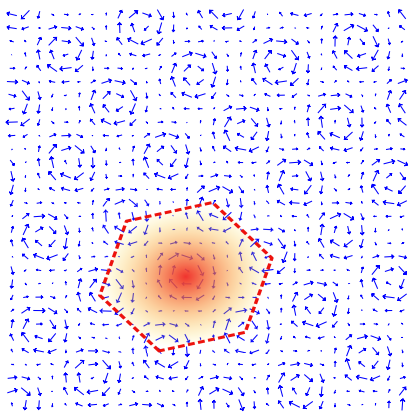

(b)

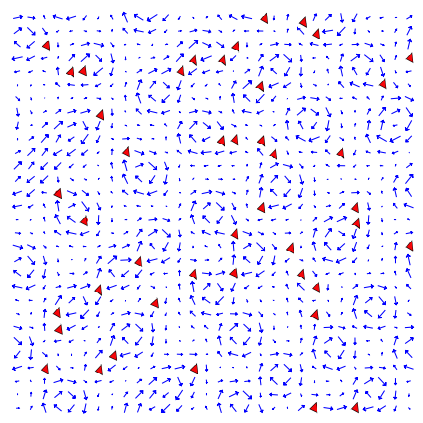

(d)
FIG. 1. (a): Spiral State in pure systems. (b): Hexagonal Skyrmion Crystal submitted to a a magnetic field $h_{z}$ and free of impurities. (c): Spiral State with 5 percent of impurities. (d): Hexagonal Skyrmion Crystal submitted to a magnetic field $h_{z}$ in a system with 5 percent of impurities.

in chiral magnets, but they also indicate that such objects should appear pinned by non-magnetic impurities throughout the whole sample, even at the absence of external field.

In this work we show that, in the presence of an impurity density $\rho$ (around a few percents), the stripe helical state undergoes a transition to a state where finite stripes show up through the sample; additionally, the skyrmion lattice state is also drastically affected, namely, its hexagonal-like collective arrangement appears to be deeply changed even at very low $\rho$. Figures 1 $(a)$ and 1(b) illustrate the ground state and the skyrmion crystal (respectively) for pure systems while Figs. 11(c) and $1(d)$ show how these same states change in a film with $5 \%$ of impurities. In more general grounds, it is known that a spin vacancy in $2 D$ nearly classical isotropic magnets generates an interaction potential that attracts the skyrmion center ${ }^{10}-12$. Hence, in these magnets containing a low impurity density, such potential allows a vacancy to be at the skyrmion center (similarly, these kind of defects also attract the vortex center in easy-plane magnets 13 and magnetic nanodisks ${ }^{14}-17$ ). Particularly, in easy-plane magnets, a low concentration of nonmagnetic impurities has a strong influence on the Berezinskii-Kosterlitz-Thouless $(B K T)$ transition temperature $\frac{18}{}$ and in magnetic nanostructures they generate the physical mechanism behinds the experimental 
observation of the Barkhauser effect $\frac{19}{20}$. Extrapolating these ideas, we could argue that possible interactions between these topological objects and lattice defects (such as spin vacancies) may distort the helical state and the skyrmion lattice, changing the skyrmion center positions in a nontrivial way. We then investigate the properties of the skyrmion crystal and the percentages of impurities necessary for completely destroying the six fold order and the skyrmion itself. For the helical state, we observe that vacancies stimulate the creation of bimerons above the perfect stripe-domain structure. Vacancies may then develop a role rather similar to thermal effects 21 and also comparable to magnetic field cooling 22 , causing the creation of excitations with elongated linelike patterns 21,22 and a kind of a skyrmion melting, even at zero temperature, for a given critical concentration of lattice defects.

\section{METHODS AND RESULTS}

In order to describe our system, we explore the magnetic phase space on the basis of a minimal lattice Hamiltonian, which is the following extended two-dimensional Heisenberg model21:

$$
\begin{aligned}
H= & -J \sum_{\vec{r}} \vec{\mu}_{\vec{r}} \cdot\left(\vec{\mu}_{\vec{r}+\hat{x}}+\vec{\mu}_{\vec{r}+\hat{y}}\right)-D \sum_{\vec{r}}\left(\vec{\mu}_{\vec{r}} \times \vec{\mu}_{\vec{r}+\hat{x}} \cdot \hat{x}\right. \\
& \left.+\vec{\mu}_{\vec{r}} \times \vec{\mu}_{\vec{r}+\hat{y}} \cdot \hat{y}\right)+A_{1} \sum_{i}\left(\left(\mu_{\vec{r}}^{x}\right)^{4}+\left(\mu_{\vec{r}}^{y}\right)^{4}+\left(\mu_{\vec{r}}^{z}\right)^{4}\right) \\
& +A_{2} \sum_{i}\left(\mu_{\vec{r}}^{x} \mu_{\vec{r}+\hat{x}}^{x}+\mu_{\vec{r}}^{y} \mu_{\vec{r}+\hat{y}}^{y}\right)-\sum_{\vec{r}} \vec{h}_{\vec{r}} \cdot \vec{\mu}_{\vec{r}} .
\end{aligned}
$$

Here $\vec{\mu}_{\vec{r}}=\mu_{\vec{r}}^{x} \hat{x}+\mu_{\vec{r}}^{y} \hat{y}+\mu_{\vec{r}}^{z} \hat{z}$ is the unit spin vector at position $\vec{r}$ and the first sum is over nearest-neighbor spins with exchange interaction strength $J>0$. The second term is the Dzyaloshinskii-Moriya interaction, where $\vec{\mu}_{\vec{r}+\hat{x}}$ and $\vec{\mu}_{\vec{r}+\hat{y}}$ represent the closest spins to $\vec{\mu}_{\vec{r}}$ in the directions $x$ an $y$ respectively. The third and fourth terms, with $A_{1}$ and $A_{2}$ constants, consist in the anisotropy of the system. The last term considers the effects of an external magnetic field $\vec{h}$ (Zeeman energy).

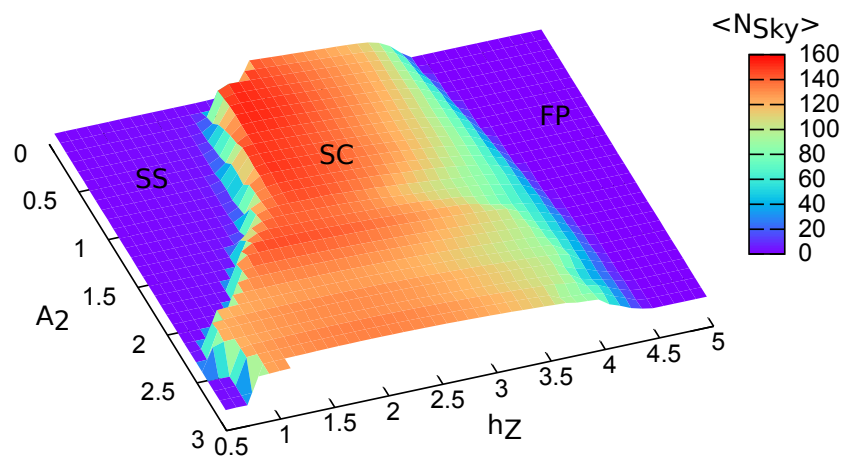

FIG. 2. Plot of the Phase diagram: Spiral State (SS), Skyrmion Crystal (SC) and Field- Polarized (FP) states.
Firstly, we consider the ground states obtained from Eq. (2). To do this, we use a simulated annealing process, which is a Monte Carlo calculation where the temperature is slightly reduced in each step of the process in order to drive the system to the global minimum. Our Monte Carlo scheme consists of a simple Metropolis algorithm. In each Monte Carlo step (MCS), we attempt to flip all spins in the lattice sequentially or randomly, which gives the same results. From an initial spin configuration obtained by randomly occupying sites with probability $1-\rho$, the simulation is performed with periodic boundary conditions for system size $N=L^{2}$; here, we have investigated lattice sizes with $L=40,60$ and 80. Then, the number of magnetically occupied sites is $N_{\text {mag }}=N(1-\rho)$. In each simulation $10^{5} \times L^{2}$ MCSs were done at each temperature, starting at $T=1.0$; the temperature is decreased in steps $\delta T=0.01$ until $T=0.01$ (the temperature is measured in units of $J / k_{B}$ ). We choose the ratio $D / J=\sqrt{6}$ (that determines the hexagonal periodicity of the skyrmion lattice) and $A_{1}=0.5$ (without loss of generality). Two techniques have been employed to measure the number of skyrmions in a state 21 : the first one takes into account the topological charge $Q$ of a skyrmion, which is the sum of the local chiral charge over the area of a single skyrmion, $Q_{s k y}=\int d^{2} x \rho_{s k y}$, with

$$
\rho_{s k y}=\frac{1}{4 \pi} \vec{\mu}_{\vec{r}} \cdot\left(\left(\vec{\mu}_{\vec{r}+\hat{x}}-\vec{\mu}_{\vec{r}-\hat{x}}\right) \times\left(\vec{\mu}_{\vec{r}+\hat{y}}-\vec{\mu}_{\vec{r}-\hat{y}}\right)\right)
$$

where $\rho_{s k y}$ is the topological charge density for several spin textures. In order to define the charge $Q$ of a skyrmion, one calculates the total charge density of the ground state and divides by the number of skyrmions. To calculate the number of skyrmions at some finite temperature one divides the total charge by $Q$. The second method 21 consists in applying $\Theta\left(1 / 2\left(\vec{\mu}_{\vec{r}} \cdot \hat{z}+1\right)-\Gamma\right)(\Theta$ is the Heaviside Theta function), were $\Gamma$ is some threshold between zero and one. Since the core of a skyrmion points against the applied magnetic field, we can binarize a state by applying the function $\Theta$ identifying spins with $\vec{\mu}_{\vec{r}} \cdot \hat{z}$ in the core of Skyrmions. By comparing these two measurements of Skyrmion number one can distinguish between reduction of topological charge due to Skyrmion destruction and alteration in of Skyrmion profile (for more details, see Ref ${ }^{21}$ ).

Before inserting impurities, we have obtained the phase diagram (for pure systems) by decreasing the temperature (in zero field) to achieve the stripe helical state structure for each $A_{2}$ (varying in the interval $0 \leq A_{2} \leq 2.0$ ). When the perpendicular external field $\vec{h}=h_{z} \hat{z}$ is applied, we vary the field in the interval $\left(0 \leq h_{z} \leq 5.0\right)$ with steps $\delta h_{z}=0.01$. The phase diagram spanned by $\left(A_{2}, h_{z}\right)$ is shown in Fig. 2 .

To start our investigation, we chose $A_{2}=0.5$ and $h_{z}=2.0$ (which are the lowest values that lead to skyrmions crystal $(S C)$ with hexagonal symmetry ) and add impurities (randomly) into the system. We find the stripe helical state with impurities and calculate both the 


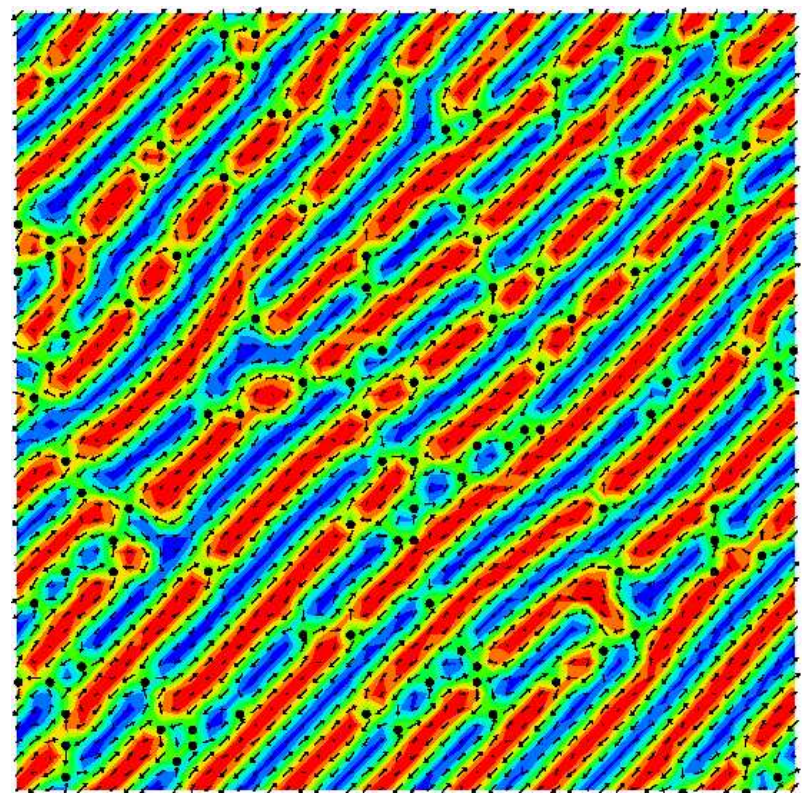

(a)

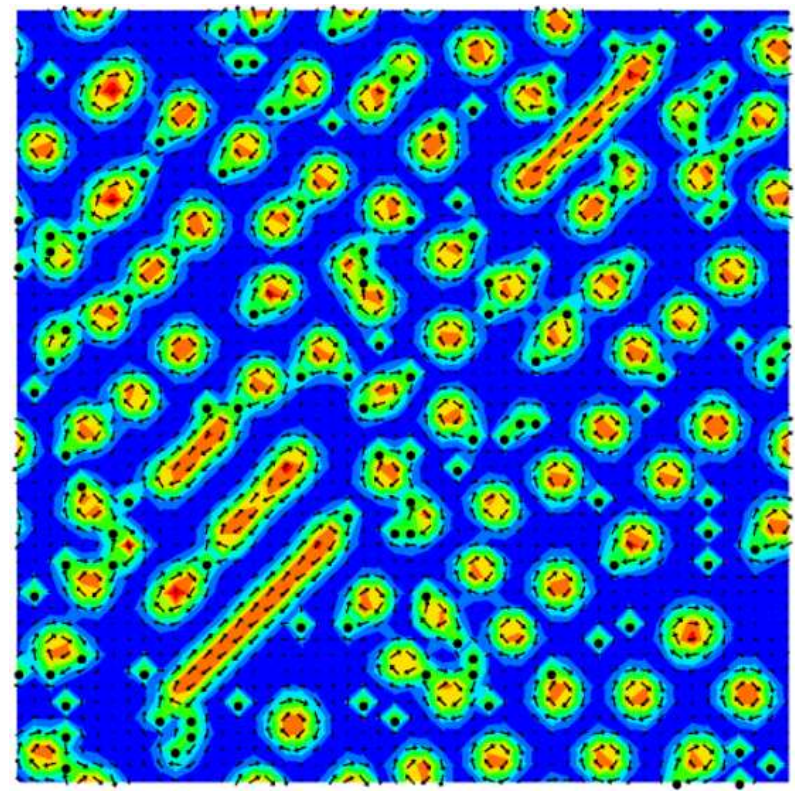

(b)

FIG. 3. (a): Spiral State with 5\% of impurities. (b): Hexagonal Skyrmion Crystal submitted to a magnetic field $h_{z}$ in a system with $5 \%$ of impurities. The background color represent the out-of-plane component of the local magnetic moment $\left(\vec{\mu}_{z}\right)$ with blue for $\mu_{z}=-1$, red for $\mu_{z}=+1$ and green for $\mu_{z}=0$. The black dots indicate the impurities positions.

number of skyrmions as a function of temperature (with and without impurities) and the number of skyrmions as a function of impurities density.

Firstly, we discuss the stripe helical state. It is interesting to note that impurities are able to create bimerons in this state. As shown in Fig 1 (c) (for a system with

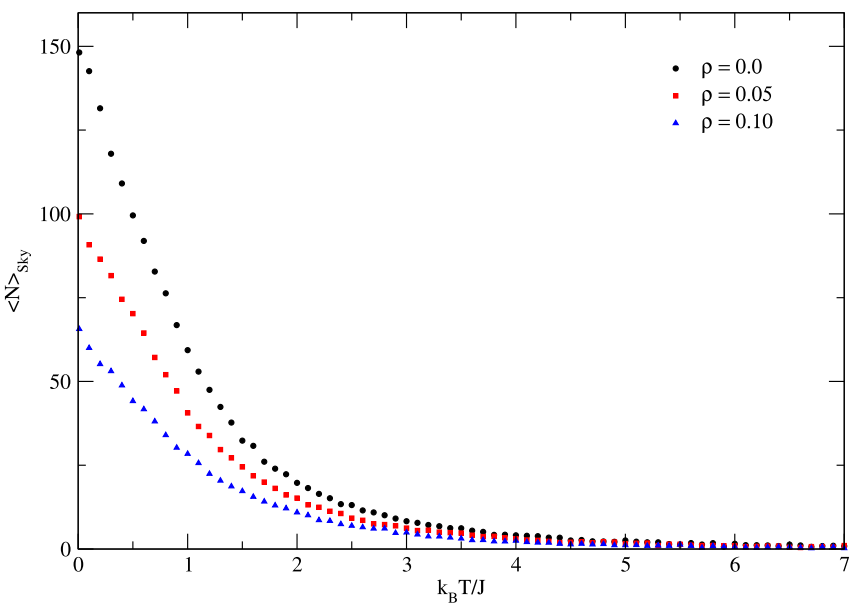

FIG. 4. Plot of the Skyrmions number $\langle N\rangle_{s k y}$ with varying $T$ for $\rho=0, \rho=0.05$ and $\rho=0.1$.

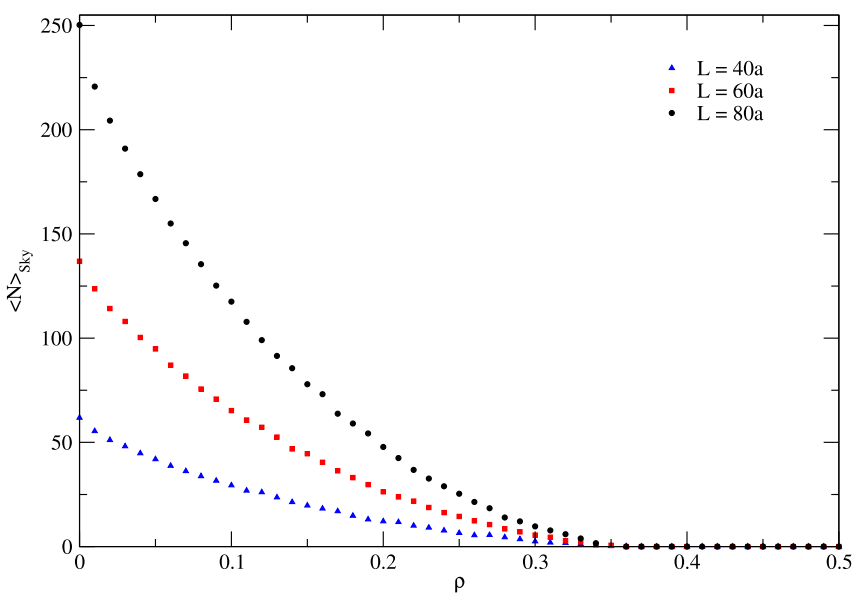

FIG. 5. Plot of the Skyrmions number $\langle N\rangle_{s k y}$ with varying impurities strength $\rho$ for $L=40 a, 60 a$ and $80 a$, at temperature $T=0.01$.

$5 \%$ of impurities), bimerons are the elongated configurations that look like a skyrmion but with its central core (which has usually the shape of a disk) shared in two half-disks separated by a rectangular stripe domain. To better see such structures, Fig. 3( $(a)$ presents the helical phase in colors: blue for spins in the negative $z$ direction $\left(S_{z}=-1\right)$, green for spins point out along the $x y$-plane $\left(S_{z}=0\right)$ and red implies $S_{z}=1$. Therefore, the helical state deformation comes about due to the presence of bimerons, which are localized between pieces of a stripe containing spin vacancies. Actually, an appreciable number of broken stripes show up even for very low impurity concentration $(\sim 1 \%)$, rendering impurities as an intrinsic mechanism to favor the appearance of bimerons in chiral magnets. Besides, impurities also offer an alternative experimental route towards their observation and even their precise localization, namely, if usual techniques, as magnetic force microscopy (MFM), is com- 
bined with magneto-mechanical setups, as those used to probe Barkhausen effect due to spin vancancies in magnetic nanostrucutures 20 . In this sense such vacancies play a role similar to magnetic field (as suggested by Ezawa 8 for pure samples, and observed in field cooling processes by Milde et $a^{22}$ ) and also to thermal effects, as found by Ambrose and Stamps 21 . Furthermore, these meron pairs will not be organized in regular lattices; they should be located around impurities randomly distributed throughout the sample. Additionally, in contrast to the behavior of the usual excitations in the $2 D$ isotropic magnets, the bimerons obtained in our simulation do not have their half-disks centered at spin vacancies; they rather localize along the diagonal direction of the $x y$-plane and they are, in general, located between two spin vacancies (in the same stripe, namely when these vacancies are separated by relatively large distances).

Now we consider the skyrmion crystal state when nonmagnetic impurities are present (see Figs. 1](c) and 3 $3(b)$ ). Figure 4 shows the dependence of the skyrmion number $\langle N\rangle_{s k y}$ as a function of temperature (considering three values of impurity concentration $\rho=0,0.05$ and 0.1). Ambrose and Stamps ${ }^{21}$ ) have studied such thermal behavior of the skyrmion lattice (and the resulting skyrmion melting) for the case in which the system is pure $(\rho=0)$. These authors have found that the thermal distortion of the skyrmion profiles (rather than destruction of the skyrmions themselves) starts to occur at relatively low temperature $\left(T \approx 0.3 J / k_{B}\right)$. At higher temperatures, there is a sharp loss of the six fold order associated with the creation of elongated structures that disrupt ordering. In what follows, we argue that the effects due to the presence of vacancies in the system lattice is somewhat similar to that of thermal effects as found in Ref. 21. Indeed, it distorts the skyrmion profiles, decreasing the skyrmion number as the impurity concentration increases and induces the presence of bimerons. We found that even at zero temperature, the number of skyrmions decreases rapidly as $\rho$ increases. To see this behavior we have also plotted the skyrmion number as a function of $\rho$ (see Fig 5 ). For a fixed very low temperature $\left(T=0.01 J / k_{B}\right)$, one can easily observe that the skyrmion number decreases monotonically down to its minimum value $\left(\langle N\rangle_{s k y}=0\right)$ achieved in a critical $\rho=\rho_{c} \approx 0.35$, where skyrmions are not present in the system anymore.

This critical value $\rho_{c}$ practically does not depend on the lattice size. One of the reasons for the reduction of the skyrmion number is that bimerons also appear in the skyrmion crystal and some of them occupy large space regions which could contain 4 or more skyrmions (there are also smaller bimerons, occupying a space of 2 or 3 skyrmions). We also notice that the six fold order is lost even for low concentration of vacancies. In fact, it is easy to see in Fig 5 that the skyrmion number reduces to almost $1 / 3$ for $\rho=0.05$ and to almost $1 / 2$ for $\rho=0.1$ (at $\left.T=0.01 J / k_{B}\right)$. Such large lost of skyrmions can not sustain the perfect six folder order along all the sys-

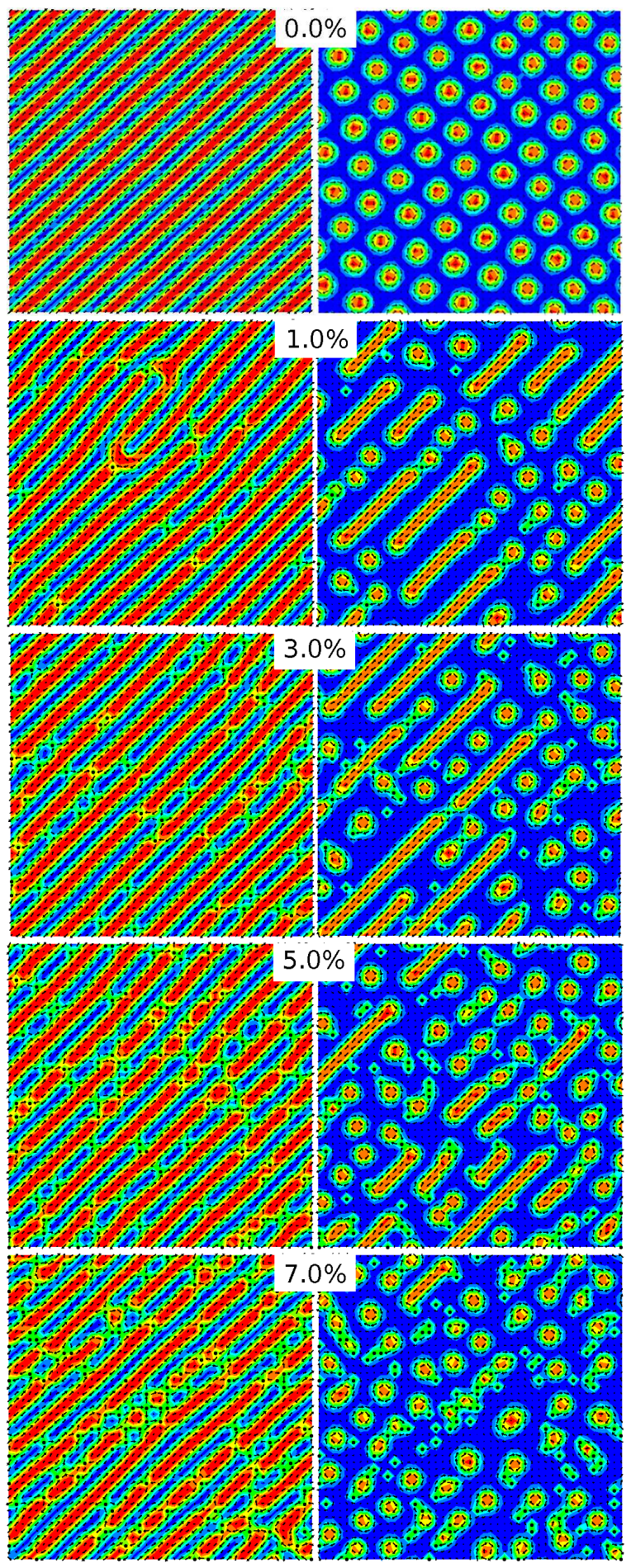

FIG. 6. The spiral and skyrmion states in a linear sequence with different concentrations of impurities.

tem. Our calculations indicate that a small concentration of impurities (less than one percent) is already sufficient for destroying the six fold order, deforming both the configuration of a large number of skyrmions and the skyrmion lattice itself. In Fig 6 we show the helical and skyrmion states in a sequence of impurities concen- 
tration. Although isolated skyrmions are attracted to the spin vacancies ${ }^{10}-12$ in isotropic $2 D$ Heisenberg magnets, which allow an impurity to be at a skyrmion center, skyrmions in a skyrmion crystal (like bimerons in the helical state) are not, in general, centered at vacancies. It is very common to see a sequence of defects containing a compact meron, an impurity and a skyrmion along a diagonal line in the $x y$-plane (see Fig 7 for the case of one percent of impurity at $\left.T=0.01 k_{B} / J\right)$. Therefore, in contrast to isolated skyrmions, energy minimization does not favor these objects to be pinned to spin vacancies when the skyrmion crystal is taken into account. There are situations (more energetically favorable) and in this case, the energy minimization must involve the whole crystal. We notice that the skyrmion lattice is formed in such a way that the spin vacancies become, in general, localized between two skyrmions or between a skyrmion and a bimeron around the system lattice. In a perfect skyrmion crystal, the spins tend to become outof-plane in the regions between two skyrmions but when impurities are present in these regions, they tend to put spins pointing along the plane directions.

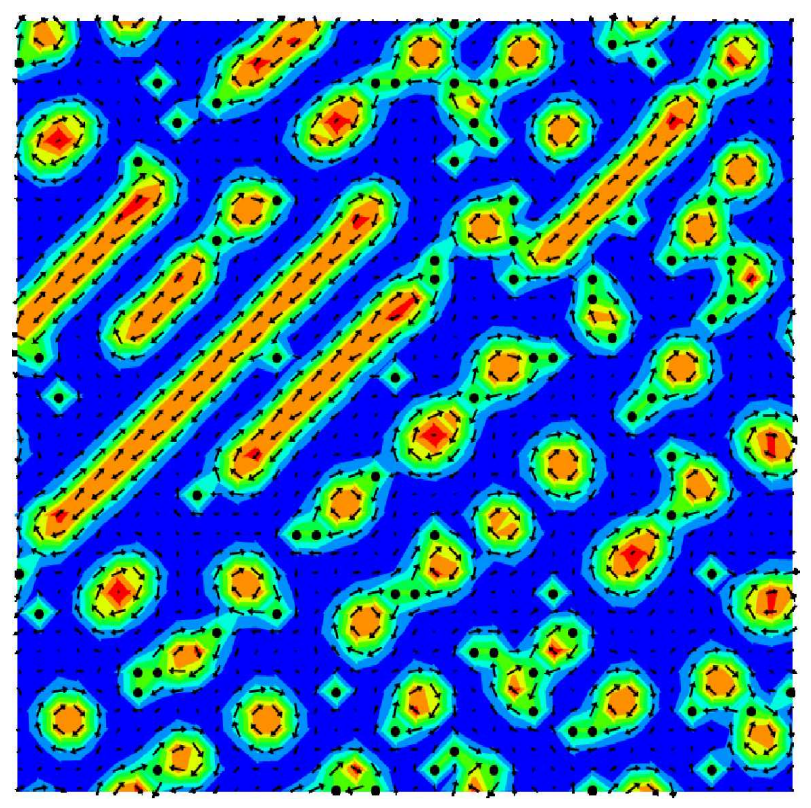

FIG. 7. Typical configuration of a $2 D$ chiral magnet containing $1 \%$ of impurities.

We have also investigated the influence of defects containing a number of adjacent impurities removed from the system. These are hexagonal-shape defects introduced in the sample plane turning it out a non-simply connected manifold. Such larger defects may be intentionally introduced into the system. We have studied the cases in which the hole has radius $\kappa$ raging from one lattice constant $a$ (four spins removed) to $9 a$ (256 spins removed). Figure 8 shows the situations for $\kappa=2 a$ and $\kappa=9 a$. In general, for small holes $(\kappa \leq 6 a)$, the six fold order is not destroyed for regions not so near the defect. Along

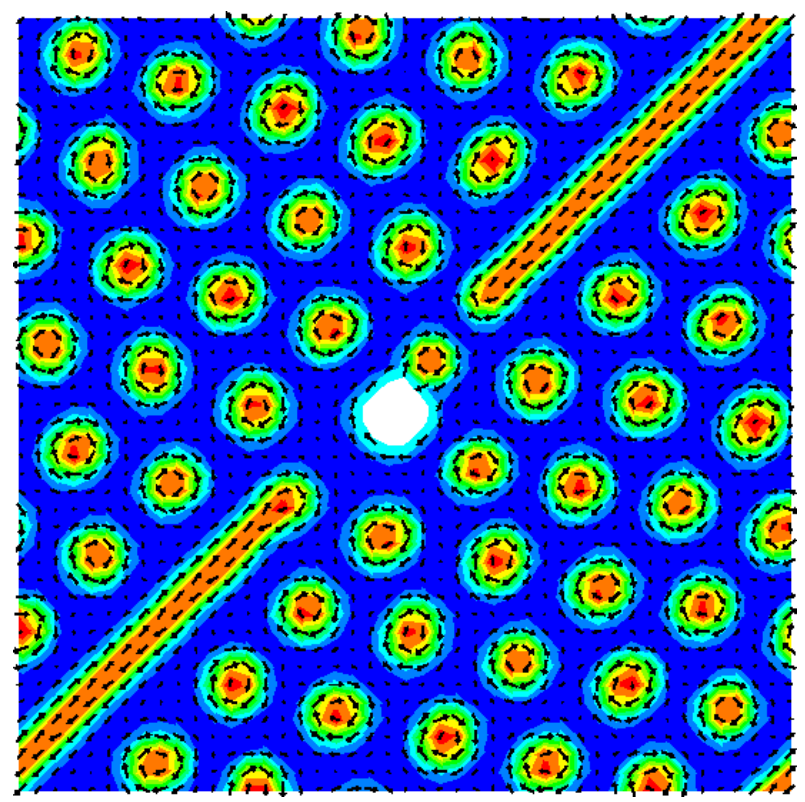

(a)

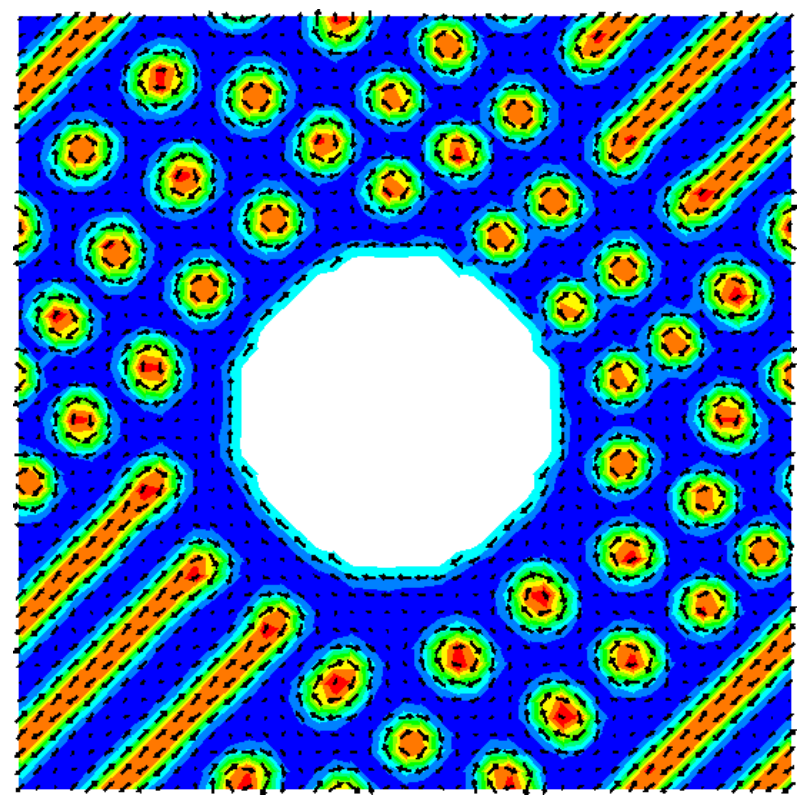

(b)

FIG. 8. (a): Typical configurations of a $2 D$ chiral magnet containing a hole in its center (some adjacent spins removed forming an almost circular defect with radius $\kappa$ ). (a): $\kappa=2 a$. (b): $\kappa=9 a$.

the diagonal line passing for the hole center, it is common to surge bimerons. Around the hole border, the spins tend to become in the plane developing a vortexlike configuration. On the other hand, for relatively large holes $(\kappa \geq 7 a)$, bimerons start to appear even along diagonal lines very apart, evidencing the nonlocal influence of such defects. The situation is similar to that obtained with impurities and external perpendicular field (see Fig. 
3(b) and related text) where skyrmion six fold order and elongated stripes (bimerons) coexist. The main novelty here is that larger defects tend to preserve skyrmions in regions around the hole.

\section{CONCLUSIONS AND PROSPECTS}

Here, we have performed Monte Carlo simulations for diluted $2 D$ chiral magnets; the helical state and the skyrmion lattice were investigated. We have found that the randomly insertion of nonmagnetic impurities into the system induces the occurrence of bimerons in the helical state (ground state) by breaking some stripedomain structures. These compact merons also surge in the skyrmion crystal phase, decreasing the number of skyrmions. Impurities distort both the skyrmion configuration and the skyrmion lattice. Therefore, spin vacancies may be faced as an intrinsic mechanism inducing phase transition from skyrmion lattice to spiral state where elongated spin stripes show up abundantly throughout the system. In this sense, they appear to work similar to thermal effects in pure materials (see Ambrose and Stamps ${ }^{21}$ ). It is also noteworthy to mention that the same overall effect is obtained by the application of suitable magnetic field, as suggested by Ezawa ${ }^{8}$, and eventually, as observed in experiments with magnetic field cooling by Milde et a $a 22$. Here, we have shown that the same happens as spin vacancies are continuously added to the system. As impurity concentration reaches a critical value $\rho_{c} \approx 0.35$ (regardless the lattice size), vestiges of skyrmion disappear completely and the system cannot support them anymore. We have also investigated how a non-simply connected manifold could affect the skyrmion crystal by considering adjacent impurities forming a large circular-type hole. All these types of defects like spin vacancies may affect several properties of the system; for instance, since a net chiral charge is present for a relatively large range of impurity density $(\rho<0.35)$ then, at a fixed temperature, the Hall conductivity may present interesting different consequences for different concentrations of impurities. As a final remark, we would like to mention that spin vacancies may offer additional advantages for probing topological objects, including skyrmions, merons, and bimerons, in chiral magnets. Actually, by combining magnetic microscopy setups with magneto-mechanical apparatus, like those used to investigate Barkhausen effects 20 , not only their direct detection as well as their precise location could be determined with very good accuracy.

\section{ACKNOWLEDGMENTS}

The authors thank CNPq and FAPEMIG for financial support.
* ricardo.l.silva@ufes.br

$\dagger$ leonardo.secchin@ufes.br

¥ wamoura.melo@gmail.com

$\S$ apereira@ufv.br

- Robert.Stamps@glasgow.ac.uk

1 T.H.R. Skyrme, Proceedings of the Royal Society of London. Series A. Mathematical and Physical Sciences. 260(1300), 127 (1961).

2 Belavin and Polyakov, JEPT Lett. 22, 245 (1975).

${ }^{3}$ F. Waldner, J. Magn. Magn. Matter. 54-57, 873 (1986).

${ }^{4}$ F. Waldner, Phys. Rev. Lett. 65, 1519 (1990).

${ }^{5}$ C.E. Zaspel, T.E. Grigereit, and J.E. Drumheller, Phys. Rev. Lett. 74, 4539 (1995).

6 X.Z. Yu, Y. Onose, N. Kanazawa, J.H. Park, J.H. Han, Y. Matsui, N. Nagaosa, and Y. Tokura, Nature 465, 901 (2010).

7 S. Heinze, K. von Bergmann, M. Menzel, J. Brede, A. Kubetzka, R. Wiesendanger, G. Bihimayer, and S. Blügel, Nature Phys. 7, 713 (2011).

8 M. Ezawa, Phys. Rev. B 83, 100408(R) (2011).

9 M. Ezawa, Phys. Rev. Lett. 105, 197202 (2010).

10 K. Subbaraman, C.E. Zaspel, and J.E. Drumheller, Phys. Rev. Lett. 80, 2201 (1998).

11 L.A. Mól, A.R. Pereira, and W.A. Moura-Melo, Phys. Rev. B 67, 132403 (2003).

12 A.R. Pereira and A.S.T. Pires, J. Magn. Magn. Mat. 257,
290 (2003).

13 A.R. Pereira, S.A. Leonel, P.Z. Coura, and B.V. Costa, Phys. Rev. B 71, 014403 (2005).

14 M. Rahm, M. Schneider, J. Biberger, R. Pulwey, J. Zweck, and D. Weiss, App. Phys. Lett. 82, 4110 (2003).

15 F.M. Paula, A.R. Pereira, and L.A.S. Mól, Phys. Lett. A 329, 155 (2004).

16 R.L. Silva, A.R. Pereira, R.C. Silva, W.A. Moura-Melo, N.M. Oliveira-Neto, S.A. Leonel, and P.Z. Coura, Phys. Rev. B 78, 054423 (2008).

17 A.R. Pereira, A.R. Moura, W.A. Moura-Melo, D.F. Carneiro, S.A. Leonel, and P.Z. Coura, J.APP. Phys. 101, 034310 (2007).

18 G.M. Wysin, A.R. Pereira, I.A. Marques, S.A. Leonel, and P.Z. Coura, Phys. Rev. B 72, 094418 (2005).

19 F.A. Apolonio, W.A. Moura-Melo, F.P. Crisafuli, A.R. Pereira, and R.L. Silva, J. App. Phys. 106, 084320 (2009).

20 J.A.J. Burgess, A.E. Fraser, F. Fani Sani, D. Vick, B.D. Hauer, J.P. Davis, and M.R. Freeman, Science 339, 1051 (2013).

21 M.C. Ambrose and R.L. Stamps, arXiv: 1209.0556v1, condmat (2012).

22 P. Milde, D. Köhler,J. Seidel,L. M. Eng, A. Bauer, A. Chacon, J. Kindervater, S. Mühlbauer, C. Pfleiderer, S. Buhrandt, C. Schütte, and A. Rosch, Science 340, 1076 (2013). 